\title{
Decreased pretherapy serum apolipoprotein A-I is associated with extent of metastasis and poor prognosis of non-small-cell lung cancer
}

This article was published in the following Dove Press journal:

OncoTargets and Therapy

\author{
Hui Shi',* \\ Haidong Huang ${ }^{1, *}$ \\ Jin $\mathrm{Pu}^{2}$ \\ Dongchen Shi' \\ Yunye Ning' \\ Yuchao Dong' \\ Yiping Han' \\ Paul Zarogoulidis ${ }^{3}$ \\ Chong Bai' \\ 'Department of Respiratory and \\ Critical Care Medicine, Changhai \\ Hospital Affiliated to The Second \\ Military Medical University, Shanghai \\ 200433, People's Republic of China; \\ ${ }^{2}$ Department of Special Clinic, \\ Changhai Hospital, Affiliated to the \\ Second Military Medical University, \\ Shanghai 200433, People's Republic \\ of China; ${ }^{3}$ Pulmonary Department, \\ Oncology Unit, "Theagenio" Cancer \\ Hospital, Thessaloniki, Greece \\ *These authors contributed equally \\ to this work
}

Correspondence: Chong Bai Department of Respiratory and Critical Care Medicine, Changhai Hospital, Affiliated to the Second Military Medical University, No 168 Changhai

Road, Shanghai 200433, People's

Republic of China

Email bc7878@sohu.com
Background: Apolipoprotein A-I (ApoA-I), which recently attracted great attention as an important protein related to the increasing risk of various cancers, is a factor closely related to metabolic diseases such as ardiovascular diseases and atherosclerosis. However, the diagnostic and prognostic value of pretherapy serum ApoA-I levels in non-small-cell lung cancer (NSCLC) patients is still not very clear.

Methods: In 325 NSCLC patients and 312 healthy controls, pretherapy serum ApoA-I was measured by turbidimetric immunoassay. The association of serum ApoA-I levels with the clinicopathologic characteristics and clinical outcomes of NSCLC patients was analyzed. Receiver-operating characteristic (ROC) curve analysis and univariate and multivariate Cox regression analyses were used to assess the diagnostic and prognostic significance of serum ApoA-I levels.

Results: Serum ApoA-I levels were obviously decreased in NSCLC patients compared with healthy controls $(1.22 \pm 0.27$ vs $1.46 \pm 0.22 \mathrm{~g} / \mathrm{L}, P<0.0001)$. Pretherapy serum ApoA-I levels were significantly decreased in the NSCLC patients with increased pretherapy C-reactive protein levels $(P=0.046)$, lower albumin serum level $(P=0.040)$, advanced TNM stage $(P=0.004)$, poorer Eastern Cooperative Oncology Group PS: performance status scores $(P=0.007)$, and more than two sites of distant metastasis $(P<0.0001)$. ROC curve showed the optimal cut-off for ApoA-I was $1.26 \mathrm{~g} / \mathrm{L}$ (Area under $\mathrm{ROC}$ curve $=0.69,95 \% \mathrm{CI}=0.54-0.65$ ) with a specificity of 0.75 and a sensitivity of 0.59 . The whole cohort was divided into two groups: low ApoA-I levels group (ApoA-I $\leq 1.26 \mathrm{~g} / \mathrm{L}$ ) consisted of 193 (59.4\%) patients and high ApoA-I levels group (ApoA-I $>1.26 \mathrm{~g} / \mathrm{L})$ consisted of $132(40.6 \%)$ patients. The median survival time of low and high ApoA-I levels patients were 16.45 and 20.90 months, respectively, which indicated a statistically significant difference $\left(\chi^{2}=0.609, P<0.0001\right)$ between the two groups. The multivariate analysis results showed that CRP levels (HR=1.273, $P=0.038)$, ApoA-I levels $(\mathrm{HR}=0.761, P=0.030)$, Eastern Cooperative Oncology Group performance status ( $\mathrm{HR}=1.486, P=0.016)$, and extent of metastasis (HR=1.394, $P=0.009)$ were significant independent predictors of favorable overall survival.

Conclusion: A decreased level of pretherapy ApoA-I was associated with a worse survival in patients with NSCLC. Serum ApoA-I measurement before initial treatment may be a novel and routine biomarker to evaluate for metastasis and predict prognosis for NSCLC patients in daily clinical practice.

Keywords: NSCLC, apolipoprotein A-I, metastasis, prognosis

\section{Introduction}

Lung cancer is the leading cause of cancer-related death worldwide. ${ }^{1}$ Despite advances in the treatment of lung cancer in recent years, the 5 -year survival rate remains $<15 \%$, attributed to the fact that $\sim 70 \%$ of lung cancer patients were diagnosed at advanced stage 
with the presence of different sites of metastatic lesions. ${ }^{2,3}$ Approximately $85 \%$ of lung cancer patients are non-smallcell lung cancer (NSCLC). Although many serum biomarkers have been reported to predict the survival of NSCLC, their sensitivity and specificity are not sufficient and reliable. ${ }^{4}$ Therefore, there is a vital task and rising demand to identify an accurate biomarker and diagnostic tool to predict the prognosis of NSCLC.

Research on lipid metabolism and cancer has gained great attention by scholars. ${ }^{5-7}$ Previous studies found that the serum high-density lipoprotein cholesterol (HDL-C) level has been shown to be a favorable prognostic factor in NSCLC ${ }^{8}$ It is reported that apolipoprotein A-I (ApoA-I) is the major polypeptide of the human plasma HDL, which comprises $\sim 70 \%$ of the HDL protein mass and consists of 243 amino acids, with molecular weight of $28.3 \mathrm{kDa} .^{9}$ ApoA-I plays a crucial role in inducing reverse cholesterol transport by combining with ATP-binding cassette transporter 1 on the walls of blood vessels and activating lecithin cholesterol acyltransferase to promote the cholesterol outflow. ${ }^{10}$ Due to its function of regulating lipid metabolism, studies on ApoA-I have long been focused in the field of atherosclerosis and cardiovascular diseases. Recently, however, the relationships between tumors and lipoproteins have attracted attention. ${ }^{11,12}$ Researchers have gradually discovered that ApoA-I plays an important role in the pathogenesis, progression, and prognosis of cancer, such as renal cell carcinoma, esophageal squamous cell carcinoma, and nasopharyngeal carcinoma. ${ }^{6,13,14}$ Although ApoA-I has been seen to correlate with worse overall survival in patients with advanced-stage NSCLC during platinum-based first-line therapy, ${ }^{15}$ the diagnostic value and prognostic significance of baseline serum ApoA-I in patients with NSCLC remain unclear. Therefore, the current study was designed to evaluate the significance of serum ApoA-I as a biomarker to predict the prognosis in patients with NSCLC.

\section{Materials and methods}

\section{Patient population and specimens}

The study group consisted of 325 confirmed cases of NSCLC at the Changhai Hospital, affiliated with the Second Military Medical University, from January 2008 to December 2014. All the included patients met the following criteria: 1) histopathologically confirmed NSCLC according to the WHO criteria; the stage classification was according to the 7th edition of Union for International Cancer Control TNM staging system; ${ }^{16}$ 2) available clinical information and laboratory data including complete ApoA-I records at diagnosis of lung cancer; 3) complete follow-up data; 4) have not received any tumor-related therapy; 5) the healthy controls consisted of 312 age-matched healthy subjects who performed a routine health check-up at Department of Special Clinic in the Changhai Hospital, affiliated to the Second Military Medical University. The exclusion criteria for patients were as follows: 1) patients who had other previous malignancy; 2) patients who were using any other medicine to treat diabetes, hyperlipidemia, metabolic syndrome, and liver disease; and 3) pregnant female and women who were taking oral contraceptives. All participants with NSCLC were followed up ranging from 1 to 60 months. This retrospective study was approved by the Institutional Review Board of the Changhai Hospital, affiliated to the Second Military Medical University and by the Ethics Committees of Changhai Hospital. Written informed consent was obtained from all involved patients.

\section{Data collection and laboratory tests}

The patients' general clinical characteristics were recorded, including their age, sex, body mass index (BMI), tobacco index, histopathological types, Eastern Cooperative Oncology Group (ECOG) performance status (PS) score, TNM stage, and medication history. Measurement of serum ApoA-I was taken at baseline and before the start of treatment. ApoA-I was determined by turbidimetric immunoassay (Sekisui Medical Co. Ltd., Tokyo, Japan) using a Hitachi 7600-110 automatic biochemical analyzer. The patients were fasting for at least 12 hours and had no alcohol intake for at least 24 hours prior to blood sampling. The patients' blood was collected in the morning between 6:00 am and 7:00 am for serum tests. Blood samples were tested in the clinical laboratory in the Changhai Hospital, affiliated to Second Military Medical University. All tests were performed according to the manufacturer's instructions. Measurement of serum ApoA-I was taken at baseline before treatment. Patients were divided into two groups according to ApoA-I kinetics to compare clinical pathological characteristics and further analyses of prognosis of patients between two groups. We used an EGFR kit (GP Medical Technologies Ltd, Beijing, China) to detect a deletion mutation in exon 19 (delE746-A750 and delT747-P753.S) and point mutation in exon 21 (L858R and L861Q) with real-time PCR.

\section{Statistical analyses}

All statistical analyses were performed using SPSS version 14.0 statistical software package (SPSS Inc., Chicago, IL, USA) and Graphpad Prism version 5.01 statistical software (Graphpad Software, Inc., CA, USA). We used median (interquartile range) to describe measurement data with a 
non-normal distribution, and the data with a normal distribution were expressed as the mean \pm SD. The unpaired Student's $t$-test and one-way ANOVA test were used to compare means between two and within several groups, respectively. Differences in continuous variables were analyzed by nonparametric tests (Mann-Whitney $U$-test or Kruskal-Wallis test). We used receiver-operating characteristic (ROC) curves to evaluate sensitivity and specificity of the serum ApoA-I and to choose its best diagnosis cut-off points to stratify patients at a high risk of malignancy-related death. Area under ROC curve (AUC), sensitivity, and specificity were counted to determine the diagnostic value of serum ApoA-I level. The score closest to the point with both maximum sensitivity and specificity was selected as the cut-off value. The cut-off score of baseline serum ApoA-I that yielded maximum AUC was $1.26 \mathrm{~g} / \mathrm{L}$. The patient population was subdivided based on the cut-off value of pretherapy serum ApoA-I as high group (ApoA-I $>1.26 \mathrm{~g} / \mathrm{L}$ ) and low group (ApoA-I $\leq 1.26 \mathrm{~g} / \mathrm{L}$ ). The chi-squared test was used to compare the differences between categorical characteristics. Survival analysis was calculated by the Kaplan-Meier method and log-rank test. Significant variables in the multivariate analysis were performed by the Cox regression analysis model. A two-sided $P<0.05$ was considered statistically significant.

\section{Results \\ Clinicopathologic characteristics in NSCLC patients and normal controls}

In this study, we included 325 patients diagnosed with NSCLC and 312 normal controls. The baseline characteristics of the study subjects are described in Table 1. There was no statistically significant difference between NSCLC patients and normal controls in age or gender. The mean age of the patients and normal controls was 60.1 and 59.2 years, respectively. The proportion of male gender for patients and normal controls was $61.8 \%$ and $59.0 \%$, respectively. The proportion of tobacco index $>400$ was higher in NSCLC patients than in normal controls ( $44.3 \%$ vs $33.3 \%$, respectively). The distribution of disease stage was as follows: stage I-II, 30.5\% (99 patients) and stage III-IV, 69.5\% (226 patients).

\section{ROC analysis of baseline serum ApoA-I levels in NSCLC patients}

The cut-off points of baseline serum ApoA-I levels in NSCLC patients were determined by ROC curve analyses. We evaluated the probability in discriminating NSCLC patients from normal controls by serum ApoA-I levels. ROC curve showed that the optimal cut-off for ApoA-I was
Table I Clinicopathologic factors in NSCLC patients and normal controls n (\%)

\begin{tabular}{|c|c|c|}
\hline Variable & $\begin{array}{l}\text { NSCLC } \\
\text { cases } \\
(n=325)\end{array}$ & $\begin{array}{l}\text { Normal } \\
\text { controls } \\
(n=3 \mid 2)\end{array}$ \\
\hline \multicolumn{3}{|l|}{ Gender } \\
\hline Male & $201(6 I .8)$ & I $84(59.0)$ \\
\hline Female & $124(38.2)$ & $128(41.0)$ \\
\hline Age (years) & $60.14 \pm 9.73$ & $59.16 \pm 11.54$ \\
\hline \multicolumn{3}{|l|}{ Tobacco index } \\
\hline$\leq 400$ & |8| (55.7) & $208(66.7)$ \\
\hline$>400$ & I 44 (44.3) & $104(33.3)$ \\
\hline \multicolumn{3}{|l|}{ BMI } \\
\hline$\leq 24 \mathrm{~kg} / \mathrm{m}^{2}$ & $192(59.1)$ & $205(65.7)$ \\
\hline$>24 \mathrm{~kg} / \mathrm{m}^{2}$ & I33 (40.9) & $107(34.3)$ \\
\hline \multicolumn{3}{|l|}{ Histological subtype } \\
\hline Adenocarcinoma & $176(54.2)$ & \\
\hline Squamous cell carcinoma & $114(35.1)$ & \\
\hline Others & $35(10.8)$ & \\
\hline \multicolumn{3}{|l|}{ Greatest tumor diameter } \\
\hline$\leq 3 \mathrm{~cm}$ & $174(53.5)$ & \\
\hline$>3 \mathrm{~cm}$ & $|5|(46.5)$ & \\
\hline \multicolumn{3}{|l|}{ Clinical stage } \\
\hline I-II & $99(30.5)$ & \\
\hline III-IV & $226(69.5)$ & \\
\hline \multicolumn{3}{|l|}{$N$ stage } \\
\hline No-2 & $248(76.3)$ & \\
\hline N3 & $77(23.7)$ & \\
\hline \multicolumn{3}{|l|}{ Distant metastases } \\
\hline$\leq 2$ sites & $207(63.7)$ & \\
\hline$>2$ sites & $118(36.3)$ & \\
\hline \multicolumn{3}{|l|}{ ECOG PS } \\
\hline $0-1$ & $276(84.9)$ & \\
\hline $2-4$ & $49(15.1)$ & \\
\hline
\end{tabular}

Abbreviations: BMI, body mass index; NSCLC, non-small-cell lung cancer; PS, performance status.

$1.26 \mathrm{~g} / \mathrm{L}(\mathrm{AUC}=0.69,95 \% \mathrm{CI}=0.54-0.65$; Figure 1$)$ with a specificity of 0.75 and a sensitivity of 0.59 . Furthermore, the whole cohort was divided into two groups: low ApoA-I levels group (ApoA-I $\leq 1.26 \mathrm{~g} / \mathrm{L}$ ), which included 193 (59.4\%) patients and high ApoA-I levels group (ApoA-I >1.26 g/L), which included $132(40.6 \%)$ patients (Table 2$)$.

\section{Association between baseline serum ApoA-I levels and clinical characteristics}

Turbidimetric immunoassay was used to investigate the pretherapy serum ApoA-I levels in patients with NSCLC and normal controls. The baseline ApoA-I level was $1.22 \pm 0.27 \mathrm{~g} / \mathrm{L}$ (range 0.36-2.10 g/L). Comparison of pretherapy ApoA-I levels between NSCLC patients and normal controls demonstrated that ApoA-I was obviously lower in NSCLC patients than in normal controls $(1.46 \pm 0.22 \mathrm{~g} / \mathrm{L}$, Figure 2A). We further analyzed the clinicopathologic significance of the pretherapy serum ApoA-I expression. 


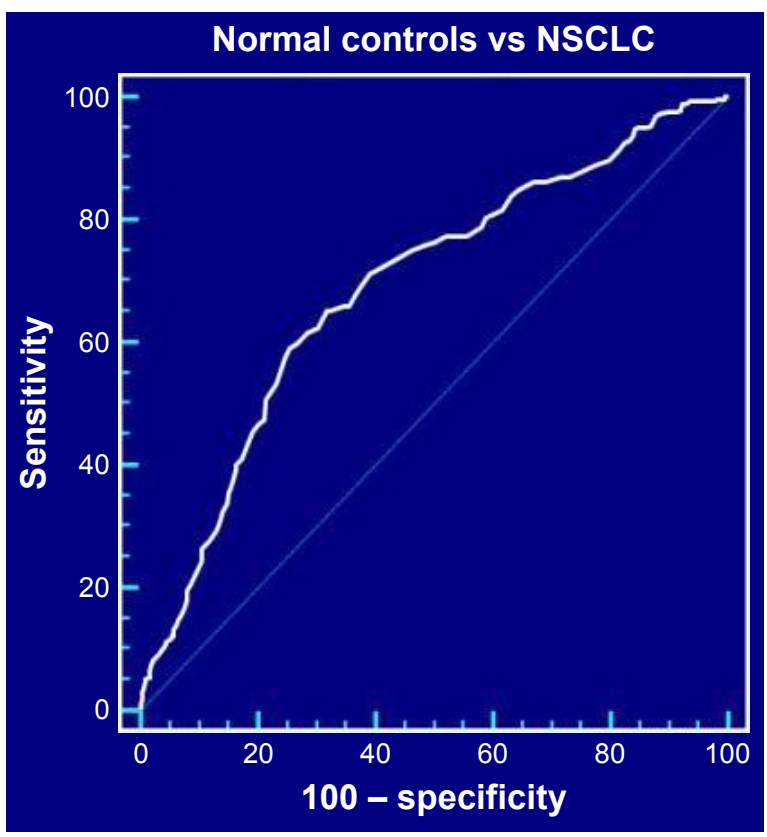

Figure I ROC analysis for pretherapy serum ApoA-I to differentiate NSCLC patients from healthy controls. Area under $R O C$ curve $(A \cup C)=0.69$, sensitivity $=0.59$, specificity $=0.75$, cutoff $=1.26$.

Abbreviations: ApoA-I, apolipoprotein A-I; NSCLC, non-small-cell lung cancer; ROC, receiver-operating characteristic.

The association between baseline serum ApoA-I levels and the clinical characteristics in NSCLC patients is summarized in Table 2. Serum ApoA-I was lower in patients with baseline serum C-reactive protein (CRP) level $>10 \mathrm{mg} / \mathrm{L}$ than in patients with baseline serum CRP level $\leq 10 \mathrm{mg} / \mathrm{L}$ (Figure 2B). Serum ApoA-I was lower in patients with baseline serum albumin level $\leq 35 \mathrm{~g} / \mathrm{L}$ than in patients with baseline serum albumin level $>35 \mathrm{~g} / \mathrm{L}$ (Figure 2C). Serum ApoA-I levels were significantly lower in NSCLC patients with advanced stage (III-IV) than those with early stage (I-II) (Figure 2D). We also analyzed the relationship between baseline serum ApoA-I levels and the extent of distant organ metastases. Results revealed that serum ApoA-I levels were significantly lower in NSCLC patients with the extent of metastasis $>2$ sites than those with the extent of metastasis $\leq 2$ sites (Figure 2E). Patients with worse ECOG PS score (2-4) were more inclined to have lower serum ApoA-I levels (Figure 2F).

The clinicopathologic features of the NSCLC cases with ApoA-I levels $\leq 1.26 \mathrm{~g} / \mathrm{L}$ were compared with those of NSCLC cases with ApoA-I levels $>1.26 \mathrm{~g} / \mathrm{L}$ (Table 2). In the entire cohort, decreased serum ApoA-I levels were significantly demonstrated in patients with increased pretherapy CRP levels $(P=0.046)$, lower albumin level $(P=0.040)$, advanced TNM stage $(P=0.004)$, more than two sites of distant metastasis $(P<0.0001)$, and poorer ECOG
PS scores $(P=0.007)$. For other clinicopathologic characteristics, ApoA-I levels were not obviously associated with age, BMI, tobacco index, EGFR mutation status, histological subtype, greatest tumor diameter, $\mathrm{N}$ classification, tumor differentiation, and chemotherapy cycles (Table 2).

\section{Univariate and multivariate analyses of serum ApoA-I levels as a prognostic factor for overall survival of NSCLC patients}

To explore the potential clinical value and to evaluate the prognostic significance of the serum pretherapy ApoA-I levels in NSCLC, clinicopathologic features were evaluated by the univariate and multivariate Cox regression models (Table 3). We excluded those patients from our statistical analyses that we could not make a proper follow-up and those that died due to a non-cancer related cause. The follow-up time ranged from 1 to 60 months. Based on the cut-off value from ROC analysis, we used the serum pretherapy ApoA-I level $1.26 \mathrm{~g} / \mathrm{L}$ as the threshold to partition the 325 NSCLC patients into two groups: high serum ApoA-I group (ApoA-I $>1.26 \mathrm{~g} / \mathrm{L}, \mathrm{n}=132$ ) and low serum ApoA-I group (ApoA-I $\leq 1.26 \mathrm{~g} / \mathrm{L}, \mathrm{n}=193$ ). As determined by KaplanMeier survival analysis and log-rank test, the lower serum ApoA-I group was correlated with a shorter overall survival $(P<0.0001$, Figure 3$)$. The median survival was 16.45 months $(95 \% \mathrm{CI}=14.73-18.17)$ for patients in high serum ApoA-I group and 20.90 months $(95 \% \mathrm{CI}=18.63-23.17)$ in low serum ApoA-I group.

The univariate analysis revealed that pretherapy serum CRP levels, albumin, clinical stage, serum pretherapy ApoA-I levels, N classification, ECOG PS, and extent of metastasis had a significant association with NSCLC survival. To determine whether these clinical variables could be used as independent prognostic factors for survival, they were subjected to multivariate Cox regression analysis. The results showed that CRP levels (HR=1.273, $P=0.038)$, ApoA-I levels (HR=0.761, $P=0.030)$, ECOG PS (HR=1.486, $P=0.016)$, and extent of metastasis ( $\mathrm{HR}=1.394, P=0.009)$ were significantly independent predictors of favorable overall survival. Thus, our findings indicate that pretherapy serum ApoA-I level may be used as a novel independent prognostic factor for NSCLC patients (Table 3).

\section{Discussion}

In this study, we investigated the correlation between pretherapy serum ApoA-I levels and clinicopathologic features in the NSCLC patients and found that ApoA-I levels were significantly lower in NSCLC patients than in healthy controls. 
Table 2 Relationship between ApoA-I and clinicopathologic factors in NSCLC patients

\begin{tabular}{|c|c|c|c|c|c|c|}
\hline \multirow[t]{2}{*}{ Variables } & \multirow{2}{*}{$\begin{array}{l}\text { Cases } \\
(n)\end{array}$} & \multirow{2}{*}{$\frac{\text { ApoA-I (g/L) }}{\text { Mean } \pm \text { SD }}$} & \multirow[t]{2}{*}{$P$-value } & \multicolumn{2}{|c|}{ ApoA-I (g/L) } & \multirow[t]{2}{*}{$P$-value } \\
\hline & & & & $\leq 1.26$ & $>1.26$ & \\
\hline \multicolumn{7}{|l|}{ Number of cases } \\
\hline Gender & & & 0.323 & & & 0.212 \\
\hline Male & 201 & $1.238 \pm 0.272$ & & 114 & 87 & \\
\hline Female & 124 & $1.210 \pm 0.270$ & & 79 & 45 & \\
\hline Age (years) & & & 0.364 & & & 0.113 \\
\hline$\leq 60$ & 165 & $1.213 \pm 0.267$ & & 105 & 60 & \\
\hline$>60$ & 160 & $1.242 \pm 0.275$ & & 88 & 72 & \\
\hline Tobacco index & & & 0.249 & & & 0.212 \\
\hline$\leq 400$ & 181 & $1.239 \pm 0.280$ & & 102 & 79 & \\
\hline$>400$ & 144 & $1.212 \pm 0.259$ & & 91 & 53 & \\
\hline BMI & & & 0.332 & & & 0.493 \\
\hline$\leq 24 \mathrm{~kg} / \mathrm{m}^{2}$ & 192 & $1.211 \pm 0.28 \mathrm{I}$ & & 117 & 75 & \\
\hline$>24 \mathrm{~kg} / \mathrm{m}^{2}$ & 133 & $1.250 \pm 0.255$ & & 76 & 57 & \\
\hline CRP & & & 0.046 & & & 0.027 \\
\hline$\leq 10 \mathrm{mg} / \mathrm{L}$ & 198 & $1.250 \pm 0.273$ & & 108 & 90 & \\
\hline$>10 \mathrm{mg} / \mathrm{L}$ & 127 & $1.191 \pm 0.264$ & & 85 & 42 & \\
\hline Albumin & & & 0.040 & & & 0.004 \\
\hline$\leq 35 \mathrm{~g} / \mathrm{L}$ & 98 & I. $182 \pm 0.289$ & & 70 & 28 & \\
\hline$>35 \mathrm{~g} / \mathrm{L}$ & 227 & $1.246 \pm 0.26 \mid$ & & 123 & 104 & \\
\hline Histological subtype & & & 0.186 & & & 0.190 \\
\hline Adenocarcinoma & 176 & $1.202 \pm 0.26 \mid$ & & 111 & 65 & \\
\hline Squamous cell carcinoma & 114 & $1.265 \pm 0.294$ & & 60 & 54 & \\
\hline Others & 35 & $1.232 \pm 0.230$ & & 22 & 13 & \\
\hline Greatest tumor diameter & & & 0.089 & & & 0.308 \\
\hline$\leq 3 \mathrm{~cm}$ & 181 & $1.248 \pm 0.259$ & & 103 & 78 & \\
\hline$>3 \mathrm{~cm}$ & 144 & $1.200 \pm 0.284$ & & 90 & 54 & \\
\hline Clinical stage & & & 0.004 & & & 0.025 \\
\hline I-II & 89 & $1.300 \pm 0.267$ & & 44 & 45 & \\
\hline III-IV & 236 & $1.200 \pm 0.268$ & & 149 & 87 & \\
\hline$N$ stage & & & 0.085 & & & 0.097 \\
\hline No-2 & 222 & $1.250 \pm 0.267$ & & 125 & 97 & \\
\hline N3 & 103 & I. $.177 \pm 0.274$ & & 68 & 35 & \\
\hline Tumor differentiation & & & 0.202 & & & 0.079 \\
\hline Well and moderately differentiated & 193 & $1.244 \pm 0.262$ & & 110 & 83 & \\
\hline Poorly differentiated & 132 & $\mathrm{I} .20 \mathrm{I} \pm 0.284$ & & 88 & 44 & \\
\hline ECOG PS & & & 0.007 & & & 0.029 \\
\hline $0-1$ & 276 & $1.243 \pm 0.269$ & & 157 & 119 & \\
\hline $2-4$ & 49 & $1.134 \pm 0.266$ & & 36 & 13 & \\
\hline Extent of metastasis & & & $<0.000$ I & & & $<0.000$ I \\
\hline$\leq 2$ sites & 207 & $1.317 \pm 0.236$ & & 98 & 109 & \\
\hline$>2$ sites & 118 & $1.069 \pm 0.255$ & & 95 & 23 & \\
\hline EGFR mutation & & & 0.734 & & & 0.877 \\
\hline Mutation & 86 & $1.261 \pm 0.226$ & & 47 & 39 & \\
\hline Wild-type & 122 & $1.244 \pm 0.283$ & & 68 & 54 & \\
\hline Chemotherapy cycles & & & 0.311 & & & 0.329 \\
\hline$<4$ & 106 & $1.200 \pm 0.294$ & & 67 & 39 & \\
\hline$\geq 4$ & 219 & $1.240 \pm 0.258$ & & 126 & 93 & \\
\hline
\end{tabular}

Abbreviations: ApoA-I, apolipoprotein A-I; BMI, body mass index; CRP, C-reactive protein; NSCLC, non-small-cell lung cancer; PS, performance status.

Pretherapy serum ApoA-I levels were associated with CRP levels, albumin, clinical stage, ECOG PS score, and extent of metastasis. Decreased serum ApoA-I levels correlated with a shorter survival period, which indicates that decreased serum
ApoA-I levels reflected poor prognosis in NSCLC patients, and Cox regression analysis revealed that serum ApoA-I levels were an independent prognostic factor for NSCLC patients. ApoA-I is synthesized predominantly in the liver and the small 

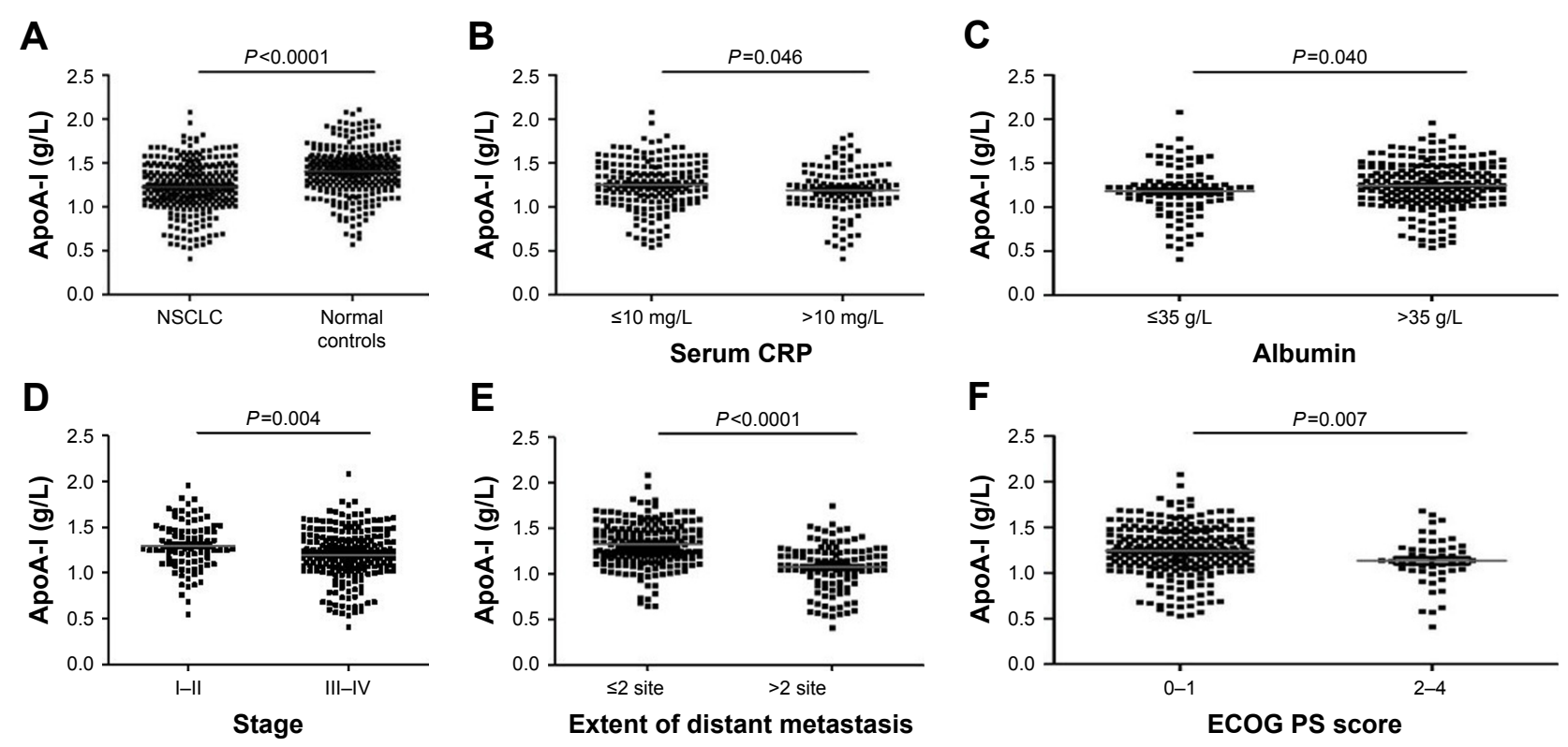

Figure 2 Comparison of serum ApoA-I levels.

Notes: (A) Between NSCLC patients and normal controls; (B) in NSCLC patients with serum C-reactive protein level $\leq 10$ and $>10$ mg/L; (C) in NSCLC patients with serum albumin level $\leq 35$ and $>35 \mathrm{~g} / \mathrm{L}$; (D) in NSCLC patients at different TNM stage; (E) in NSCLC patients with extent of distant metastasis $\leq 2$ sites and $>2$ sites; (F) in NSCLC patients with different ECOG PS score.

Abbreviations: ApoA-I, apolipoprotein A-I; NSCLC, non-small-cell lung cancer; PS, performance status.

intestine, which is the predominant protein of plasma HDL. ${ }^{17}$ ApoA-I is principally in charge of assembly of HDL particles to converting cholesterol to cholesteryl ester through lecithin cholesterol acyltransferase binding to ApoA-I on HDL. It is described and summarized by researchers that AopA-I/ HDL had the function of anti-inflammation, antioxidant, and antiapoptotic activities. ${ }^{18,19}$ Recently, a large meta-analysis showed that for every $10 \mathrm{mg} / \mathrm{dL}$ increase in HDL levels, there was a $36 \%$ lower risk of developing cancer. A protective role for AopA-I in various cancers was noted in several large clinical observational studies where an inverse relationship between lower serum AopA-I levels and increased risk of

Table 3 Prognostic factors for overall survival in univariate and multivariate Cox analyses

\begin{tabular}{|c|c|c|c|c|c|c|}
\hline \multirow[t]{2}{*}{ Variable } & \multicolumn{3}{|c|}{ Univariate analysis } & \multicolumn{3}{|c|}{ Multivariate analysis } \\
\hline & HR & $95 \% \mathrm{Cl}$ & $P$-value & HR & $95 \% \mathrm{Cl}$ & $P$-value \\
\hline Gender (male vs female) & 1.083 & $0.863-1.358$ & 0.493 & & & \\
\hline Age ( $\leq 60$ vs $>60$ years $)$ & 1.096 & $0.880-1.366$ & 0.413 & & & \\
\hline Tobacco index ( $\leq 400$ vs $>400)$ & 0.937 & $0.750-1.172$ & 0.570 & & & \\
\hline $\mathrm{BMI}(\leq 24$ vs $>24)$ & 0.976 & $0.780-1.221$ & 0.829 & & & \\
\hline $\mathrm{CRP}(\leq 10$ vs $>10 \mathrm{mg} / \mathrm{L})$ & 1.289 & $1.029-1.615$ & 0.027 & 1.273 & $1.014-1.599$ & 0.038 \\
\hline Albumin ( $\leq 35$ vs $>35 \mathrm{~g} / \mathrm{L})$ & 1.368 & $1.077-1.738$ & 0.010 & 1.242 & $0.970-1.590$ & 0.086 \\
\hline Histological subtype & 1.037 & $0.883-1.217$ & 0.658 & & & \\
\hline \multicolumn{7}{|l|}{$\begin{array}{l}\text { Adenocarcinoma (squamous cell carcinoma vs } \\
\text { adenosquamous carcinoma vs others) }\end{array}$} \\
\hline Greatest tumor diameter ( $\leq 3$ vs $>3 \mathrm{~cm})$ & 0.850 & $0.681-1.061$ & 0.850 & & & \\
\hline ApoA-I ( $\leq \mathrm{I} .26$ vs $>1.26 \mathrm{~g} / \mathrm{L})$ & 0.609 & $0.485-0.765$ & $<0.0001$ & 0.761 & $0.594-0.975$ & 0.030 \\
\hline Clinical stage (I-II vs III-IV) & 1.320 & $1.029-1.692$ & 0.029 & 1.206 & $0.936-1.554$ & 0.148 \\
\hline $\mathrm{N}$ stage (N0-2 vs N3) & 1.365 & $1.078-1.730$ & 0.010 & 1.245 & $0.979-1.584$ & 0.074 \\
\hline Tumor differentiation & 1.146 & $0.913-1.439$ & 0.241 & & & \\
\hline \multicolumn{7}{|c|}{ (Well and moderately differentiated vs poorly differentiated) } \\
\hline ECOG PS (0-I vs $2-4)$ & 1.658 & $|.222-2.25|$ & 0.001 & 1.486 & $1.077-2.05 \mid$ & 0.016 \\
\hline Extent of metastasis ( $\leq 2$ sites vs $>2$ sites) & $\mathrm{I} .575$ & $1.247-1.989$ & $<0.0001$ & 1.394 & $1.088-1.788$ & 0.009 \\
\hline EGFR mutation (mutation vs wild-type) & 1.266 & $0.952-1.683$ & 0.104 & & & \\
\hline Chemotherapy cycle $(<4$ vs $\geq 4)$ & 1.246 & $0.986-1.575$ & 0.066 & & & \\
\hline
\end{tabular}

Abbreviations: ApoA-I, apolipoprotein A-I; BMI, body mass index; CRP, C-reactive protein; PS, performance status. 


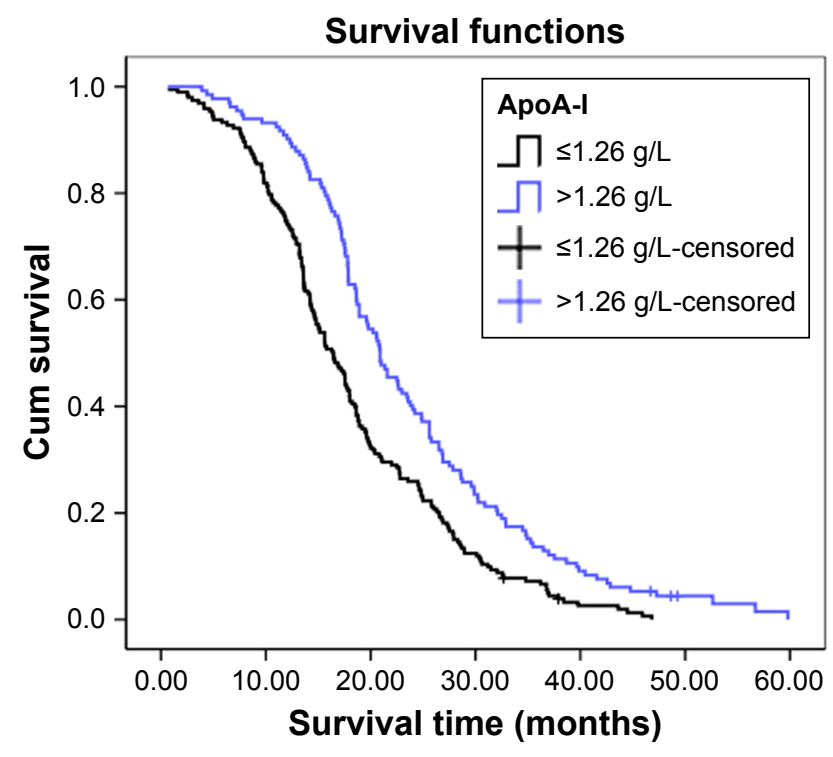

Figure 3 Kaplan-Meier survival curves of 325 NSCLC patients. Note: Overall survival rate of NSCLC patients with lower serum ApoA-I group was significantly shorter than those with higher serum ApoA-I group. Abbreviations: ApoA-I, apolipoprotein A-I; cum, cumulative; NSCLC, non-smallcell lung cancer.

developing cancer was also revealed..$^{20}$ High AopA-I mRNA levels in primary diagnosis prechemotherapy effusions were demonstrated to be significantly related with the better overall survival in advanced ovarian cancer. ${ }^{21}$ Lower serum AopA-I levels even had a practical function to predict the recurrence of breast cancer. ${ }^{22}$ Similarly, our study showed that a low baseline serum ApoA-I level is strongly related to poor overall survival time and is an independent prognostic factor for survival in NSCLC patients (HR=0.761, 95\% CI $=0.594-0.975$ ).

The exact mechanisms of the relationship between decreased serum AopA-I levels and poor survival in cancer patients have not been fully explained. Nevertheless, several potential explanations of ApoA-I antitumorigenic activity have been presented by researchers. It is known that disorders of lipid and cholesterol homeostasis facilitate the proliferation and evasion of apoptosis by tumor cells. ${ }^{23,24}$ Meanwhile, a recent study has revealed that ApoA-I potently suppresses tumor growth and metastasis in multiple animal tumor models through both innate and adaptive immune processes. ${ }^{25}$ ApoA1-deficient (A1KO) mice exhibited increased tumor burden and development of metastasis with a worse survival time. In contrast, mice expressing the human ApoA1 transgene (A1Tg) showed increased infiltration of macrophages with antitumor phenotype, which helps in preventing tumor growth and enhances survival. ${ }^{26}$ In addition, inflammation can contribute to cancer progression by producing different bioactive molecules to the tumor microenvironment, which can stimulate cancer progression. ${ }^{27}$
Cancer cells secrete extra inflammatory mediators, which promote systemic inflammatory response interactions and further lead to cell injury and related oxidative stress. Previous studies have investigated the relationship between proinflammatory cytokines such as CRP level, IL-6, and lipids. IL-6 is recognized as a master regulator of tumor-associated inflammation and tumorigenesis. ${ }^{28}$ Consistent with previous studies, our results also showed that patients with elevated CRP level had a poor overall survival. Our study also demonstrated that pretherapy ApoA-I levels are associated with the CRP levels. Overexpression of secretory nonpancreatic phospholipase A2, which is stimulated by IL-6, has been reported to decrease the concentration of ApoA-I. ${ }^{29}$ Since there is a strong connection between IL-6 level and hepatic production of CRP, the decreased ApoA-I level might be partially attributed to the elevation of CRP levels and used as indirect measure of the tumor activity.

Similar to the previous research, our data demonstrated the significant prognostic value of ECOG PS in the NSCLC patients. Moreover, a correlation between decreased serum ApoA-I level and poorer ECOG PS (2-4) was found in our study. Weaker general physical condition is always related with poorer ECOG PS score (2-4), which generally accompanies hypoalbuminemia. So it can be better understood that ApoA-I levels were found to be lower in patients with albumin $\leq 35 \mathrm{~g} / \mathrm{L}$ before initial treatment. Furthermore, clinical TNM stage and extent of metastasis have also been explored with lung cancer patients in our study. Pretherapy serum ApoA-I levels were found to be lower in patients with more organ sites of distant metastasis compared with those with less than two sites of metastasis. We also observed that elevated ApoA-I levels were related to an earlier TNM stage in NSCLC patients. Recent study has revealed that ApoA-I has been related to the metastasis and angiogenesis by changing the tumor microenvironment. Decreased matrix metalloproteinase-9 protein levels and enzyme activity combined with extracellular matrix remodeling promote tumor invasion and tumor metastasis, which might provide a reasonable explanation for this phenomenon. ${ }^{17}$

Here, some important implications might be drawn from our study for clinical practice. First, serum ApoA-I level was lower in NSCLC patients than in healthy controls. Meanwhile, through ROC/AUC analysis, we found pretherapy serum ApoA-I levels at $1.26 \mathrm{~g} / \mathrm{L}$ may be the best cut-off value for NSCLC patients. As an indicator of clinical diagnostic biomarker, it was suitable and convenient for tumor patients in routine examination. Second, we analyzed the relationship between the pretherapy serum ApoA-I level and clinical characteristics and discovered 
that decreased pretherapy ApoA-I levels were associated with advanced TNM stage, ECOG PS, extent of metastasis, CRP, and albumin. Furthermore, ApoA-I could function as an independent prognostic factor for NSCLC patients.

Several limitations existed in our study. First, more prospective randomized, multicentric studies were needed to validate our conclusions. Second, chemotherapeutic targeted therapy effect together with other serum cholesterol data, such as value of HDL and low-density lipoprotein, should be added to improve evaluation results.

\section{Conclusion}

Compared to other laboratory examinations, ApoA-I measurement, before initial treatment, is available and inexpensive and may provide a novel and routine biomarker to evaluate metastasis and predict prognosis for NSCLC patients in daily clinical practice.

\section{Acknowledgments}

This study was supported by the projects of Shanghai Zhangjiang National Independent Innovation Demonstration Zone Special Development Fund Major Project (grant number: 201505-YP-A101-002) and the Shanghai Science and Technology Committee Medical Guidance Project Fund (grant number: 134119a0302).

\section{Disclosure}

The authors report no conflicts of interest in this work.

\section{References}

1. Herbst RS, Morgensztern D, Boshoff C. The biology and management of non-small cell lung cancer. Nature. 2018;553(7689):446-454.

2. Jones CM, Brunelli A, Callister ME, Franks KN. Multimodality treatment of advanced non-small cell lung cancer: where are we with the evidence? Curr Surg Rep. 2018;6(2):5.

3. de Ruysscher D, Faivre-Finn C, Le Pechoux C, Peeters S, Belderbos J. High-dose re-irradiation following radical radiotherapy for non-smallcell lung cancer. Lancet Oncol. 2014;15(13):e620-e624.

4. Xu-Welliver M, Carbone DP. Blood-based biomarkers in lung cancer: prognosis and treatment decisions. Transl Lung Cancer Res. 2017;6(6): 708-712.

5. van Hemelrijck M, Walldius G, Jungner I, et al. Low levels of apolipoprotein A-I and HDL are associated with risk of prostate cancer in the Swedish AMORIS study. Cancer Causes Control. 2011;22(7):1011-1019.

6. Luo XL, Zhong GZ, Hu LY, Ly H, et al. Serum apolipoprotein A-I is a novel prognostic indicator for non-metastatic nasopharyngeal carcinoma. Oncotarget. 2015;6(41):44037-44048.

7. Wang L, Chi PD, Chen H, Xiang J, Xia ZJ, Zhang YJ. Low level of highdensity lipoprotein cholesterol correlates with poor prognosis in extranodal natural killer/T cell lymphoma. Tumour Biol. 2014;35(3):2141-2149.

8. Chi PD, Liu W, Chen H, et al. High-density lipoprotein cholesterol is a favorable prognostic factor and negatively correlated with C-reactive protein level in non-small cell lung carcinoma. PLoS One. 2014;9(3): e91080.

9. Davidson WS, Thompson TB. The structure of apolipoprotein A-I in high density lipoproteins. J Biol Chem. 2007;282(31):22249-22253.
10. Pollard RD, Fulp B, Samuel MP, Sorci-Thomas MG, Thomas MJ. The conformation of lipid-free human apolipoprotein A-I in solution. Biochemistry. 2013;52(52):9470-9481.

11. Kuzu OF, Noory MA, Robertson GP. The role of cholesterol in cancer. Cancer Res. 2016;76(8):2063-2070.

12. Tamura T, Inagawa S, Hisakura K, Enomoto T, Ohkohchi N. Evaluation of serum high-density lipoprotein cholesterol levels as a prognostic factor in gastric cancer patients. J Gastroenterol Hepatol. 2012; 27(10):1635-1640.

13. Guo S, He X, Chen Q, et al. The effect of preoperative apolipoprotein A-I on the prognosis of surgical renal cell carcinoma: a retrospective large sample study. Medicine. 2016;95(12):e3147.

14. Wang XP, Li XH, Zhang L, et al. High level of serum apolipoprotein A-I is a favorable prognostic factor for overall survival in esophageal squamous cell carcinoma. BMC Cancer. 2016;16:516.

15. Cheng T, Dai X, Zhou DL, Lv Y, Miao LY. Correlation of apolipoprotein A-I kinetics with survival and response to first-line platinum-based chemotherapy in advanced non-small cell lung cancer. Med Oncol. 2015;32(1):407.

16. Groome PA, Bolejack V, Crowley JJ, et al. The IASLC Lung Cancer Staging Project: validation of the proposals for revision of the T, N, and $\mathrm{M}$ descriptors and consequent stage groupings in the forthcoming (seventh) edition of the TNM classification of malignant tumours. J Thorac Oncol. 2007;2(8):694-705.

17. Michalaki V, Koutroulis G, Syrigos K, Piperi C, Kalofoutis A. Evaluation of serum lipids and high-density lipoprotein subfractions (HDL2, HDL3) in postmenopausal patients with breast cancer. Mol Cell Biochem. 2005;268(1-2):19-24.

18. Zamanian-Daryoush M, Didonato JA. Apolipoprotein A-I and cancer. Front Pharmacol. 2015;6:265.

19. Borgquist S, Butt T, Almgren $P$, et al. Apolipoproteins, lipids and risk of cancer. Int J Cancer. 2016;138(11):2648-2656.

20. Jafri H, Alsheikh-Ali AA, Karas RH. Baseline and on-treatment highdensity lipoprotein cholesterol and the risk of cancer in randomized controlled trials of lipid-altering therapy. J Am Coll Cardiol. 2010; 55(25):2846-2854.

21. Kozak KR, Amneus MW, Pusey SM, et al. Identification of biomarkers for ovarian cancer using strong anion-exchange ProteinChips: potential use in diagnosis and prognosis. Proc Natl Acad Sci U S A. 2003; 100(21):12343-12348.

22. Lane DM, Boatman KK, Mcconathy WJ. Serum lipids and apolipoproteins in women with breast masses. Breast Cancer Res Treat. 1995; 34(2):161-169.

23. Kucharska-Newton AM, Rosamond WD, Schroeder JC, et al. HDLcholesterol and the incidence of lung cancer in the Atherosclerosis Risk in Communities (ARIC) study. Lung Cancer. 2008;61(3):292-300.

24. von Eckardstein A, Hersberger M, Rohrer L. Current understanding of the metabolism and biological actions of HDL. Curr Opin Clin Nutr Metab Care. 2005;8(2):147-152.

25. Sharma S, Umar S, Potus F, et al. Apolipoprotein A-I mimetic peptide 4F rescues pulmonary hypertension by inducing microRNA-193-3p. Circulation. 2014;130(9):776-785.

26. Zamanian-Daryoush M, Lindner D, Tallant TC, et al. The cardioprotective protein apolipoprotein A1 promotes potent anti-tumorigenic effects. J Biol Chem. 2013;288(29):21237-21252.

27. Cruz PM, Mo H, Mcconathy WJ, Sabnis N, Lacko AG. The role of cholesterol metabolism and cholesterol transport in carcinogenesis: a review of scientific findings, relevant to future cancer therapeutics. Front Pharmacol. 2013;4:119.

28. Asegaonkar SB, Asegaonkar BN, Takalkar UV, Advani S, Thorat AP. $\mathrm{C}$-reactive protein and breast cancer: new insights from old molecule. Int J Breast Cancer. 2015;2015:145647.

29. Tietge UJ, Maugeais C, Cain W, et al. Overexpression of secretory phospholipase $\mathrm{A}(2)$ causes rapid catabolism and altered tissue uptake of high density lipoprotein cholesteryl ester and apolipoprotein A-I. J Biol Chem. 2000;275(14):10077-10084. 
OncoTargets and Therapy

\section{Publish your work in this journal}

OncoTargets and Therapy is an international, peer-reviewed, open access journal focusing on the pathological basis of all cancers, potential targets for therapy and treatment protocols employed to improve the management of cancer patients. The journal also focuses on the impact of management programs and new therapeutic agents and protocols on

patient perspectives such as quality of life, adherence and satisfaction. The manuscript management system is completely online and includes a very quick and fair peer-review system, which is all easy to use. Visit http://www.dovepress.com/testimonials.php to read real quotes from published authors.

Submit your manuscript here: http://www.dovepress.com/oncotargets-and-therapy-journal 